\title{
PKC $\alpha$ is a Potentially Useful Marker for Planning Individualized Radiotherapy for Nasopharyngeal Carcinoma
}

This article was published in the following Dove Press journal: Cancer Management and Research

\author{
Jing Zhang' \\ Lu Zhang ${ }^{2}$ \\ Bowen $\mathrm{Xie}^{2}$ \\ Yumei Duan ${ }^{3}$ \\ Ying Wang ${ }^{4}$ \\ Liangfang Shen' \\ 'Department of Oncology, Xiangya \\ Hospital, Central South University \\ (CSU), Changsha, 4I0008, People's \\ Republic of China; ${ }^{2}$ Key Laboratory of \\ Molecular Radiation Oncology, Changsha, \\ Hunan Province, 410008, People's \\ Republic of China; ${ }^{3}$ Department of \\ Pathology, Xiangya Hospital, CSU, \\ Changsha, 410008, People's Republic of \\ China; ${ }^{4}$ Department of Radiology, \\ Xiangya Hospital, CSU, Changsha, \\ 410008, People's Republic of China
}

Correspondence: Liangfang Shen Department of Oncology, Xiangya Hospital, Central South University (CSU), No. 87 Xiangya Road, Changsha, 410008, Hunan, People's Republic of China Email Ifshen2008@I63.com
Purpose: To examine the expression of protein kinase $\mathrm{C}$ alpha $(\mathrm{PKC} \alpha)$ in nasopharyngeal carcinoma (NPC) and determine its relationship to the radio-sensitivity of NPC in order to evaluate its potential as a molecular marker for the guidance of individualized radiation therapy for NPC.

Materials and Methods: PKC $\alpha$ expression levels were detected in tumor samples from patients and in NPC cell lines with varying degrees of radio-sensitivity. A survival analysis was performed to analyze the association of PKCa expression with the 5-year overall survival (OS), progression-free survival (PFS), locoregional recurrence-free survival (LRFS), and distant metastasis-free survival (DMFS) in patients. In vitro and in vivo experiments using NPC cell lines were performed to study the effects of down-regulation of PKC $\alpha$ by short hairpin RNA treatment on the radio-sensitivity of NPC.

Results: PKC $\alpha$ expression was up-regulated in the well-differentiated NPC tissues of patients and in the more radio-resistant NPC cell lines. Moreover, high PKC $\alpha$ expression was associated with a worse 5-year PFS and LRFS of patients. shRNA-mediated knockdown of PKCa led to an increase in the sensitivity of NPC cells to radiation therapy, both in vitro as cultured cells and in vivo as tumor xenografts.

Conclusion: The elevated expression of PKC $\alpha$ in NPC and its association with patient PFS indicates that $\mathrm{PKC} \alpha$ is a potential molecular marker for guiding precision radiotherapy in NPC patients. Also, the increased radiosensitivity of NPC cells after loss of PKC $\alpha$ identifies $\mathrm{PKC} \alpha$ as a promising therapeutic target for enhancing the radio-sensitivity of NPC.

Keywords: $\mathrm{PKC} \alpha$, marker, individualized radiotherapy, nasopharyngeal carcinoma

\section{Introduction}

Nasopharyngeal carcinoma (NPC) is a malignancy in the mucous epithelium of the nasopharyngeal tissues, and its prevalence shows a distinct geographic distribution. It is primarily endemic in Southeast Asia and the southern provinces of China, with a much higher incidence in these areas than in any other country or region in the world. ${ }^{1}$ Radiation therapy has been the first-line standard treatment for NPC, ${ }^{2}$ with some patients receiving intensity modulated radiotherapy (IMRT) and others receiving chemoradiotherapy. Although the overall survival (OS) of NPC patients has improved with advances in these combination radiotherapy treatments, for patients in whom these treatments fail to achieve local control, retreatment is often ineffective and causes serious complications. Because it is currently impossible to accurately predict the efficacy of radiation therapy for individuals, oncologists do not 
have a clear strategy for personalizing the treatment approach for individual NPC patients and, thus, are left to apply similar treatment regimens in all NPC cases. Therefore, research is urgently needed to identify markers that indicate which cases are likely to be more resistant to radiation therapy, as this information would allow for the planning of more individualized treatments. ${ }^{3}$

Protein kinase $\mathrm{C}(\mathrm{PKC})$ is a kinase that is widely expressed in human tissues, and PKC-alpha (PKC $\alpha)$, a subtype of PKC, has been closely associated with cancer cell proliferation, differentiation, and apoptosis. ${ }^{4}$ We previously explored the relationship between PKC $\alpha$ and local invasion of NPC and found that the PKC $\alpha$ expression level correlates with the 2-year progression-free survival (PFS) and overall survival (OS) of NPC patients. ${ }^{5}$ In the present study, we extended this research in NPC patients, investigating $\mathrm{PKC} \alpha$ expression in NPC specimens and analyzing the correlation of PKC $\alpha$ expression with the 5-year survival of NPC patients. To better understand the observed correlation, we evaluated the expression of PKC $\alpha$ in NPC cell lines with varying degrees of radio-resistance and studying the effects of $\mathrm{PKC} \alpha$ knockout on their response to radiation treatment both as cultured cells in vitro and as tumor xenografts in vivo. Together, the results from our analysis of patient specimens and our in vitro and in vivo experiments provide the evidence that $\mathrm{PKC} \alpha$ represents a potential marker of NPC radio-sensitivity as well as a potential target for increasing the radio-sensitivity of NPC.

\section{Materials and Methods NPC Clinical Samples}

NPC samples were collected from a total of 97 patients at initial diagnosis in the Oncology Department of Xiangya Hospital of Central South University between 2010 and 2015. The ethical committee of Xiangya Hospital approved this study (No: 201703513). Use of tissue samples, data collection and review of medical records were performed in accordance with the ethical standards of the committee and the 1964 Declaration of Helsinki and its later amendments. Because of the retrospective nature of our study and lack of patient identification, informed consent was waived for all participants involved in this study. The inclusion criteria included nasopharyngeal carcinoma diagnosed for the first time by biopsy and treated with radical intensity-modulated conformal radiotherapy. Patients were excluded according to the following criteria: performance status $\geq 2$ on the Eastern Cooperative Oncology Group (ECOG) scale, distant metastasis (stage $\mathrm{IVb}$ ), previous malignancy or other type of tumor; unstable cardiac disease; and current lactation or pregnancy. The nasopharyngeal tumors were paraffinembedded and formalin-fixed. The samples were collected from untreated patients along with clinical follow-up data on the initial diagnosis. The histological classifications followed the World Health Organization (WHO) classification system: type I, keratinizing carcinomas; type II, differentiated subtype of non-keratinizing carcinoma; and type III, undifferentiated subtype of non-keratinizing carcinoma.

\section{Clinical Staging Method, Image Assessment, and Treatment}

Patients' computed tomography (CT) and magnetic resonance imaging (MRI) scans were independently reviewed with the Varian Eclipse Plan System by two radiation oncologists. Stage was determined by comprehensively evaluating the patients' clinical manifestations, physical examination results, and CT or MRI scans according to Seventh Edition of the American Joint Committee on Cancer (AJCC) clinical staging standard. Measurable disease was defined by the RECIST criteria. According to the No. 50 and 62 documents of the ICRU, all patients were treated by IMRT, with prescribed doses as follows: primary tumor, 70.62-73.92 Gy (2.14-2.24 Gy/33 fractions); high-risk lymph nodes, $69.96 \mathrm{~Gy}$ (2.12 Gy/33 fractions); intermediate-risk sites, 59.4-61.05 Gy (1.8-1.85 Gy/33 fractions), and low-risk sites, 50.4-51.8 Gy (1.8-1.85 Gy/28 fractions). The radiation was delivered by a linear accelerator (Varian, USA) with five daily fractions per week. Paclitaxel/docetaxel and/or platinum were used as neo-adjuvant, concurrent, and adjuvant chemotherapy. OS was defined as the duration from the first day of initial diagnosis to the date of death from any cause or the last follow-up on August 31, 2020. Progression-free survival (PFS) and locoregional recurrence-free survival (LRFS), and distant metastasis-free survival (DMFS) were defined as the interval from the first day of initial diagnosis to confirmed disease progression, local recurrence, and distant metastasis, respectively. Progressive disease was defined as at least a $20 \%$ increase in the sum of the diameters of target lesions (an absolute increase of at least $5 \mathrm{~mm}$ ) or the appearance of one or more new lesions. Stable disease was defined as neither sufficient shrinkage 
to qualify as partial response nor sufficient growth to qualify as progressive disease. ${ }^{6}$

\section{Immunohistochemical Staining}

All xenograft samples and NPC tissue blocks were fixed and embedded in paraffin according to standard procedures. Following de-paraffinization, sections were subjected to heat-mediated antigen retrieval in $0.01 \mathrm{~mol} / \mathrm{L}$ citrate buffer ( $\mathrm{pH}$ 6.0). After cooling to room temperature, the samples were treated with $1 \%$ methanol $/ 30 \% \mathrm{H}_{2} \mathrm{O}_{2}$ to block endogenous peroxidase activity. Nonspecific protein binding was blocked using $5 \%$ bovine serum albumin (BSA) solution. Next, sections were rinsed with phosphate-buffered saline (PBS) and incubated with antiPKC $\alpha$ (Santa Cruz Biotechnology, 1:40) overnight at $4^{\circ}$ C. The next day, the sections were rinsed with PBS at room temperature and incubated with horseradish peroxidase (HRP)-conjugated secondary antibodies (Abcam, UK) for $30 \mathrm{~min}$. Then, after 3-5 min of washing, the sections were incubated with 3,3'-diaminobenzidine (DAB) for visualization. Finally, the samples were rinsed with distilled $\mathrm{H}_{2} \mathrm{O}$ and counterstained with hematoxylin.

The scores for PKC $\alpha$-reactive NPC cells in patient samples were determined by evaluating the staining degree and percentage of positive cells. For cases of positive cytoplasmic staining, PKC $\alpha$ scores were assigned as follows: 0 , absence of expression or expression in $<10 \%$ of NPC-positive cells; 1, staining in 10-49\% of NPC-positive cells; and 2, staining in $\geq 50 \%$ of NPC-positive cells. The immunohistochemical staining results were independently evaluated by two pathologists.

\section{Cell Culture and Cell Line Generation} The NPC cell lines CNE1, CNE2, and HK1 were donated by the Cancer Research Institute of Central South University. ${ }^{7-9}$ The use of the cell lines was approved by the ethics committee of Xiangya Hospital, and the CNE1 and CNE2 cells were authenticated by STR profile before the study. A radio-resistant cell line, denoted as CNE2-resistant (CR) cells, was established via exposure of parental CNE2 cells to gradually increasing doses of irradiation. ${ }^{10,11}$ The final surviving CNE2 cells were verified with significant radiation-resistant characteristics and defined as radioresistant CNE2 cells (CR) ${ }^{10}$ The radiation was delivered with a Varian linear accelerator (2100EX, San Francisco, CA, USA). The cell culture flask was covered with a $1.5-\mathrm{cm}$ layer of compensation glue during irradiation. In addition, a $6-\mathrm{MeV}$ electron beam was chosen, and the radiation dose rate was $300 \mathrm{cGy} / \mathrm{min}$. All cell lines were cultured at $37^{\circ} \mathrm{C}$ in $5 \% \mathrm{CO}_{2}$ and grown in Roswell Park Memorial Institute (RPMI) 1640 medium (Gibco, USA) with 10\% fetal bovine serum (FBS; Gibco, USA) and 1\% penicillin/ streptomycin (Invitrogen, USA). Cells in the exponential growth phase were used in the experiments.

\section{Plasmid Construction and Transfection}

To knockdown PKC $\alpha$, two target sequences were selected: the human PKC $\alpha$ target 1 (5-GGGATCGAACAACAAG GAATG-3) and target 2 (5-GCGTCCTGTTGTATGA AATGC-3). ${ }^{12}$ To generate PKC $\alpha$ short hairpin (sh)RNA, the coding sequences for the shRNAs were cloned using the Lenti-X ${ }^{\mathrm{TM}}$ shRNA Expression System (Clontech). Then 293T cells were co-transfected with lentiviral vectors, envelope plasmids, and packaging plasmids for $24 \mathrm{~h}$ before collection of the lentiviral supernatant. The CR and CNE1 cells were transduced with control or shPKC $\alpha$ lentiviral particles for $48 \mathrm{~h}$ and then cultured for $72 \mathrm{~h}$ with $2 \mu \mathrm{g} / \mathrm{mL}$ puromycin for selection. The efficacy of transfection was verified by Western blot analysis.

\section{Colony Formation and Radio-Sensitivity Assays}

Cells were seeded in six-well plates and subjected to irradiation (IR) treatment for $24 \mathrm{~h}$ with a dose range $0-6$ Gy delivered with the Varian linear accelerator $(2100 \mathrm{EX}$, San Francisco, California, USA). Our preliminary experiment showed that $6 \mathrm{~Gy}$ is an appropriate dose for further experiments. The medium was changed every 5 days after radiation, and after 10-12 days, the cells were washed three times at room temperature, fixed in paraformaldehyde for $15 \mathrm{~min}$, and stained with crystal violet for $10 \mathrm{~min}$. After washing with de-ionised water, the plates were left to air dry. For calculation of colony formation, clones with more than 50 cells were counted as colonies. Analysis was performed using the linear quadratic model of GraphPad software.

\section{Flow Cytometry}

Cells were seeded in six-well plates, irradiated with $2 \mathrm{~Gy}$ $16 \mathrm{~h}$ after seeding, and harvested at $48 \mathrm{~h}$. Then $1 \times 10^{4}$ cells were treated by Annexin V-FITC Apoptosis Detection Kit (Beyotime, China), and apoptosis was analyzed by flow cytometry (Guava easyCyte, Millipore, Germany). 


\section{Preparation of Proteins and Western Blotting}

For protein extraction, cells were lysed with radioimmunoprecipitation assay (RIPA) buffer for $30 \mathrm{~min}$ on ice. Protein concentrations were then measured with BCA protein assay kits (Thermo-Scientific, USA). Protein samples (40-50 $\mu \mathrm{g}$ ) were prepared and separated by a sodium dodecyl sulfatepolyacrylamide gel electrophoresis (SDS-PAGE) and then transferred onto 0.22 - and $0.45-\mu \mathrm{m}$ polyvinylidene difluoride (PVDF) membranes (Millipore, USA). The membranes were immersed in Tris-buffered saline with Tween 20 (TBST) containing 5\% non-fat dry milk, shaken at room temperature for 1 $\mathrm{h}$, and then incubated for $1 \mathrm{~h}$ at room temperature in solutions of the following primary antibodies at $4^{\circ} \mathrm{C}$ for $16-18 \mathrm{~h}$ : antiPKCa (1:500, Santa Cruz Biotechnology, USA) and antitubulin (1:1000, Santa Cruz Biotechnology). After washing with TBST, the blots were incubated with the secondary antibodies (1:1000 Santa Cruz Biotechnology) for $1 \mathrm{~h}$ at room temperature. Finally, the protein bands were visualised with a Thermo Scientific-Pierce Enhanced Chemiluminescence (ELC) substrate detection system.

\section{NPC Xenograft Model}

The animal experiments were conducted according to the Guidelines of the Animal Handling and Care in Medical Research Committee of Central South University and were approved by the Animal Ethics Committee of Central South University (No: 201703512). Six- to eight-week-old female nude mice obtained from Vital River Laboratories (Beijing, the original breeding pairs were purchased from Charles River) were used to establish the human NPC xenograft model. After stable transduction with PKC $\alpha$ shRNA or shControl lentivirus, $5 \times 10^{6} \mathrm{CR}$ cells were inoculated into the left flanks of the mice to form the NPC xenografts. Tumor volume was measured every 2 days from the third day after subcutaneous inoculation. Irradiation treatment was applied once the tumor volume reached $60-80 \mathrm{~mm}^{3}$. Radiation was delivered at 2 Gy per fraction every other day for three fractions. When the tumor volume reached a maximum of $1500 \mathrm{~mm}^{3}$, the mice were euthanized, and the xenografts were harvested for further analyses. ${ }^{13}$

Apoptosis in xenografts was detected by terminal deoxynucleotidyl transferase dUTP nick end labeling (TUNEL) staining using the ApopTag ${ }^{\circledR}$ Fluorescein In-Situ Apoptosis Detection Kit (Merck Millipore, Germany) after preparation of sections for staining as described above for human NPC specimens. The fluorescence intensity was analysed by ImageJ (National Institutes of Health, USA).

\section{Statistical Analysis}

All in vitro and in vivo experiments were performed at least three times. The statistical analyses were conducted using SPSS 23.0 software (SPSS, Inc., USA). For comparison of data between two groups, Student's $t$ test or chi-square test was used for in vitro data. Spearman rank correlation coefficients were used to determine the correlations of PKC $\alpha$ and histological classification. Survival was estimated using the Kaplan-Meier method, and the results were compared with the Log rank test. P values $<0.05$ were considered statistically significant. All statistical tests were two-sided.

\section{Results}

\section{Expression of PKC $\alpha$ is Up-Regulated in Well-Differentiated NPC}

Tumor samples were obtained from 97 patients with histologically diagnosed NPC. The patients ranged in age from 22-72 years and had WHO types I, II, or III NPC (stages II to Iva; Table 1). All patients exhibited a performance status of less than 2 on the Eastern Cooperative Oncology Group (ECOG) scale.

It is generally accepted in radiation oncology that poorly differentiated malignant tumours tend to be more sensitive to radiation therapy. As WHO type I is rare in NPC, we detected and analyzed PKC $\alpha$ expression in NPC specimens including WHO type II and III, considering WHO type II NPC to be well differentiated and WHO type III NPC to be poorly differentiated. Based on the expression level score derived from immunohistochemical staining analysis of PKC $\alpha$ (Figure 1A), a rank correlation analysis revealed that $\mathrm{PKCa}$ was differentially expressed

Table I Characteristics of NPC Patients Whose Samples Were Used for Immunohistochemical Staining of PKC $\alpha$

\begin{tabular}{|l|l|l|}
\hline \multicolumn{2}{|l|}{ Characteristic } & $\mathbf{n}$ \\
\hline Age & $\geq 47$ yrs & 49 \\
& $<47$ yrs & 48 \\
\hline Sex & Male & 66 \\
& Female & 3 I \\
\hline Clinical stage & II & 3 \\
& III & 35 \\
& IVa & 59 \\
\hline Histologic type & WHO I & I \\
& WHO II & 26 \\
& WHO III & 68 \\
& Not evaluated by WHO criteria & 2 \\
\hline
\end{tabular}




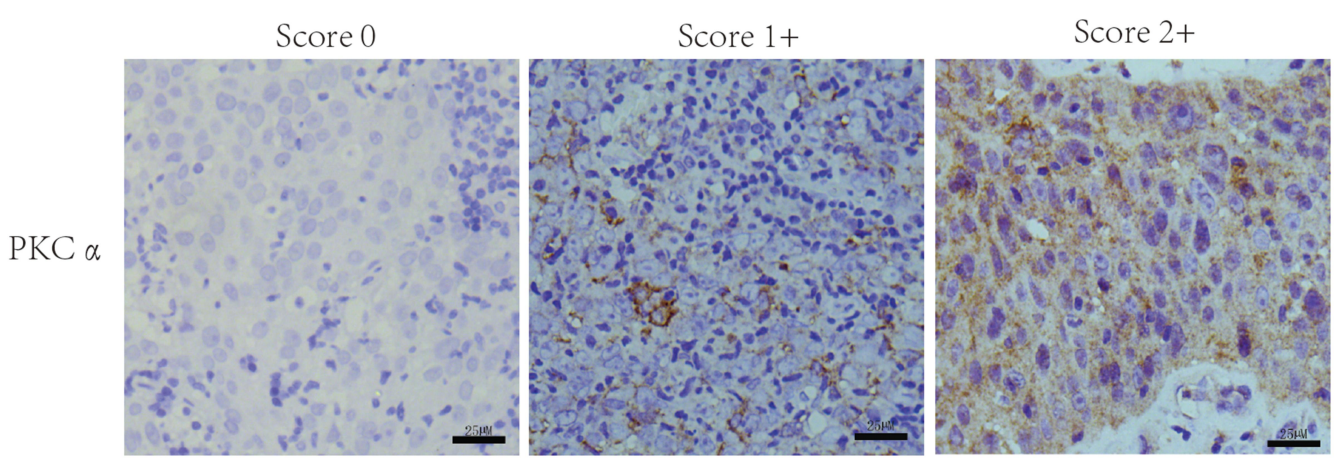

Figure I Expression of PKC $\alpha$ in NPC tissues. Representative images of PKC $\alpha$ staining in pathological specimens of NPC. The criteria for scores of 0 (left column), I+ (middle column) and $2+$ (right column) are described in the Materials and Methods (scale bar $=25 \mu \mathrm{m}$ ).

between the WHO II and WHO III groups (Spearman correlation coefficient $0.290 ; \mathrm{p}=0.005$; Table 2).

\section{PKC $\alpha$ Expression is Associated with 5-Year} Progression-Free Survival and LRFS in NPC Patients To further confirm whether PKC $\alpha$ expression is related to the survival outcomes of radiation therapy for NPC, we divided the NPC cases into a PKC $\alpha$ expression-positive group and $\mathrm{PKC}$ expression-negative group, according to pathological scores for PKC $\alpha$ staining ( 0 , negative; and score 1 and 2, positive). From a survival analysis based on the 5-year follow-up, the OS rates of the PKC $\alpha$-negative and $\mathrm{PKC} \alpha$-positive groups were $97.2 \%$ and $86.5 \%$, respectively ( $\mathrm{p}=0.084$; Figure $2 \mathrm{~A}$ ). The PFS rate of the PKC $\alpha$ negative group was significantly higher than that of the of the PKC $\alpha$-positive group $(91.7 \%$ vs $77.0 \%, p=0.045$; Figure 2B). The LRFS rate of the PKC $\alpha$-negative was also significantly higher than that of the PKC $\alpha$-positive group $(94.4 \%$ vs $80.3 \%, \mathrm{p}=0.043$; Figure $2 \mathrm{C})$. Finally, the DMFS rates of the $\mathrm{PKC} \alpha$-negative and $\mathrm{PKC} \alpha$-positive groups did not differ significantly $(94.4 \%$ vs $85.2 \%$, $\mathrm{p}=0.139$; Figure 2D).

\section{PKC $\alpha$ Expression is Up-Regulated in Radiation-Insensitive NPC Cell Lines}

Given that radiation therapy is the primary treatment for NPC and our results above suggest that PKC $\alpha$ expression is related to patients' PFS, we examined the level of PKC $\alpha$ expression in NPC cell lines that exhibit varying degrees of radio-sensitivity. First, to test radio-sensitivity, colony formation by NPC cell lines was assessed after irradiation at 0 or 6 Gy (Figure 3A). Then PKC $\alpha$ expression in these cell lines was detected by Western blotting. CR cells, which are considered largely radio-resistant (Figure S1A and $\mathrm{B}$ ), showed significantly greater $\mathrm{PKC} \alpha$ expression compared with CNE2 cells. Also CNE1 cells, a cell line with rather poor response to radiation, also showed elevated PKC $\alpha$ expression. In sharp contrast, in the radiationsensitive cell line HK1, PKC $\alpha$ expression was extremely low (Figure 3B).

\section{Reduced PKC $\alpha$ Expression Increases the Radio-Sensitivity of NPC Cells in vitro}

To investigate whether $\mathrm{PKC} \alpha$ regulates the radiosensitivity of NPC cells, we performed loss-of-function assays using two independent shRNAs targeting PKC $\alpha$. As shown in Figure 4A and B, shRNA treatment using two target sequences sh\#1 and sh\#2 resulted in successful down-regulation of PKC $\alpha$ in both the CR and CNE1 cell lines. We then examined the survival fractions of $\mathrm{CR}$ and CNE1 cells after 6 Gy of irradiation with or without PKC $\alpha$ knockdown. As shown in Figure 4C and D, knockdown of PKC $\alpha$ significantly increased the radio-sensitivity of CR and CNE1 cells compared with control cells.

Table 2 Correlation Between the Intensity of PKCa Expression in Nasopharyngeal Carcinoma (NPC) and Histological Classification

\begin{tabular}{|l|l|l|l|l|l|}
\hline & PKC $\alpha-$ & PKC $\boldsymbol{\alpha}$ I+ & PKC $\mathbf{\alpha 2 +}$ & Spearman's Rank Correlation Coefficient & P-value \\
\hline WHO II & 6 & 5 & 15 & 0.290 & $0.005 * *$ \\
\hline WHO III & 30 & 22 & 16 & & \\
\hline
\end{tabular}

Note: $* * p<0.01$. 

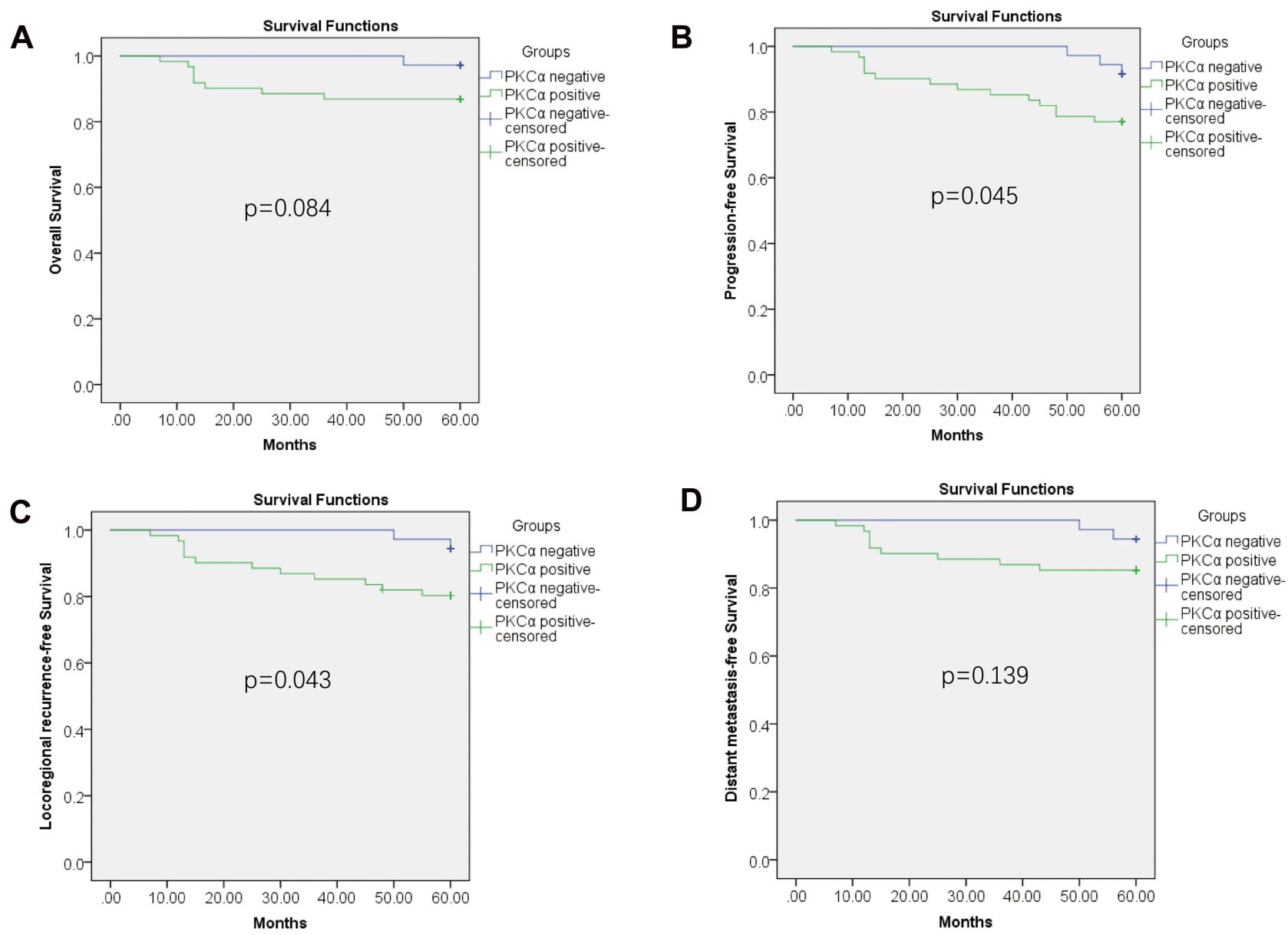

Figure 2 Association of PKC $\alpha$ expression with survival among NPC patients. (A) Comparison of 5-year OS between NPC patients in the PKC $\alpha$-negative and -positive groups. (B) Comparison of 5-year PFS between NPC patients in the PKC $\alpha$-negative and -positive groups. (C) Comparison of 5-year LRFS between NPC patients in the PKC $\alpha$-negative and -positive groups. (D) Comparison of 5-year DMFS between NPC patients in the PKC $\alpha$-negative and -positive groups.

\section{Reduced PKC $\alpha$ Expression Increases the Radio-Sensitivity of CR Xenografts in vivo}

Next, we established a mouse NPC xenograft model using $\mathrm{CR}$ cells to investigate whether knockdown of PKC $\alpha$ could enhance NPC sensitivity to radiation in vivo. After subcutaneous inoculation of shPKC $\alpha$ - or scramble control-treated CR cells into nude mice, the two types of NPC cells formed tumors well. From day 7 to day 11 ( 5 days), we treated mice three times, once every other day, with 2-Gy fractions. Consistent with the results in vitro, tumor growth in the shPKC $\alpha$ group was significantly inhibited in response to radiation treatment, compared with that in the control groups (Figures 5A, B and $\underline{\mathrm{S} 2}$ ). Immunohistochemical staining of harvested xenografts confirmed down-regulation of PKC $\alpha$ in the shPKC $\alpha$-treated group (Figure 5C). Additionally, more apoptotic tumor cells were observed in the xenografts from the shPKC $\alpha$ group after radiation treatment (Figure 5D and
E). Together, these results indicate that down-regulation of PKC $\alpha$ can increase the sensitivity of CR cells within NPC xenografts to radiation therapy.

\section{Discussion}

It is generally accepted that PKC functions as a carcinogenic promoter; ${ }^{14}$ however, decreased activity and expression levels of several subtypes of PKC also have been found in many malignant tumors. ${ }^{15}$ These seemingly conflicting findings reveal the complexity of the cellular functions of PKC and its subtypes in cancer development. ${ }^{16}$

Studies have also reported that PKC expression is related to therapeutic resistance in cancer cases, ${ }^{17}$ and $\mathrm{PKC} \alpha$ expression has been suggested to be a predictor of tumor recurrence after treatment. ${ }^{18,19}$ In primary human fibroblasts, PKC signaling was shown to prevent

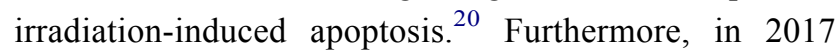
Scott et al proposed a gene expression-based radiation- 
A
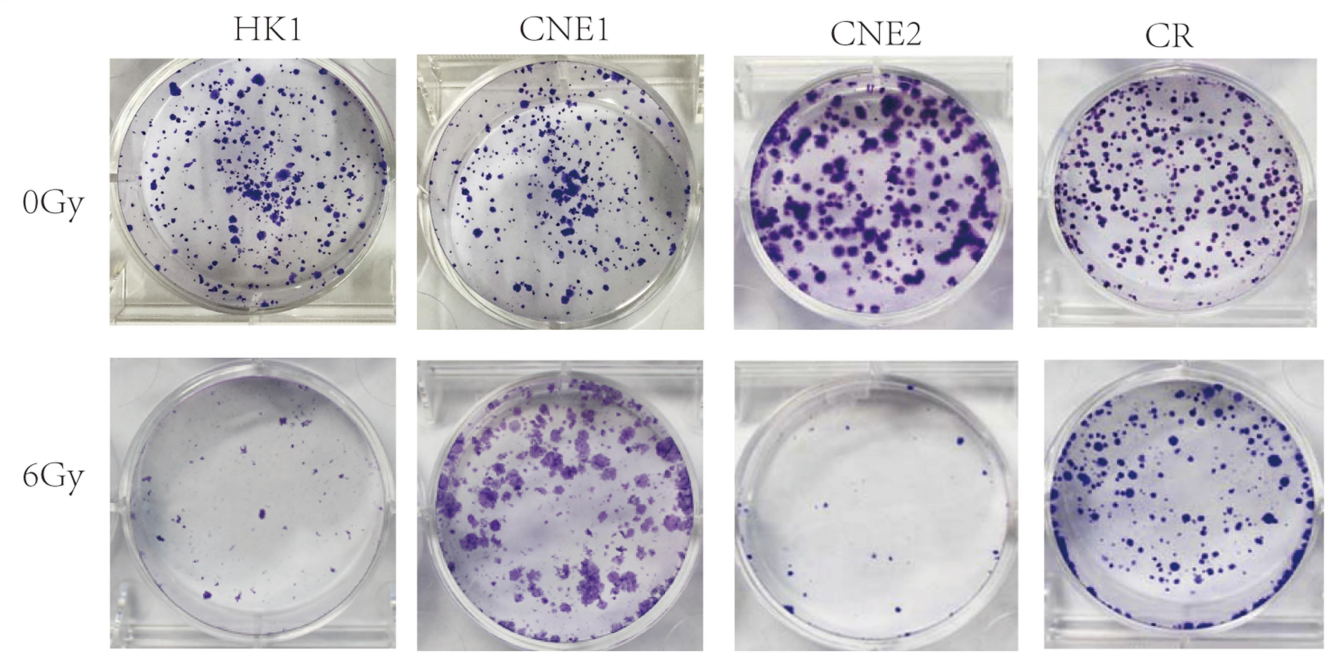

B

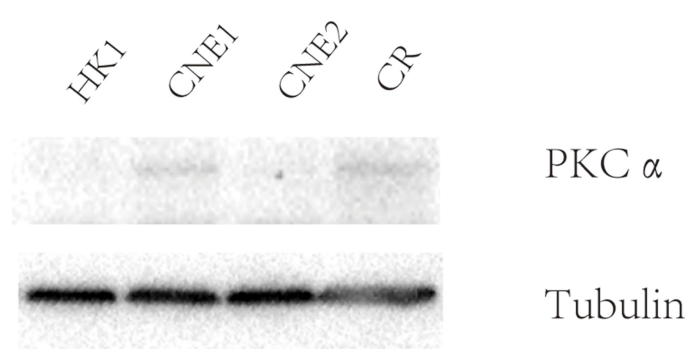

Figure 3 PKC $\alpha$ expression in NPC cell lines. (A) Clonogenic assay of HKI, CNE2, CNEI and CR cells after exposure to 0 and 6 Gy radiation $(n=3 ; P<0.05)$. (B) Western blotting analysis of PKC $\alpha$ expression in HKI, CNEI, CNE2 and CR cells, Tubulin was used as a loading control.

sensitivity index that includes $\mathrm{PKC} \beta$ expression for planning an individualized radiation dose for NPC. ${ }^{21}$ These studies indicate that the PKC signaling pathway is involved in the regulation of resistance to therapy and, specifically, radio-resistance. Therefore, the results of our present study confirm the link between PKCa expression and radio-resistance and provide evidence for the potential value of PKC $\alpha$ as a molecular marker for guiding individualized radiation therapy for NPC. As a marker in NPC, high PKC $\alpha$ expression would indicate the need for a relatively high dose of radiation therapy to improve the likelihood of local disease control and thereby improve patients' survival. It is foreseeable that under the guidance of individualized PKCa levels, radiation oncologists will be able to make radiation treatment plans more individualized. At the same time, comprehensive treatment plans including chemotherapy could be optimized to avoid excessive treatment. In addition, in view of the results of the present study, the significance of PKC $\alpha$ expression in the WHO classification deserves further exploration.

The main types of NPC are WHO type II and III, and WHO type I NPC, which is also defined as keratinizing carcinomas, is extremely rare. In our study, PKCa expression in WHO type II cases was higher than that in type III cases, which is consistent with previous conclusion that PKC is related to the differentiation of normal cells and malignant tumors. ${ }^{22-24}$ However, the NPC-specific mechanism of PKC $\alpha$ in the regulation of cancer cell differentiation requires further investigation.

A limitation of this study was the inability to determine the relationship between the expression of PKC $\alpha$ and the immunohistochemical markers of differentiation in order to understand more clearly whether PKC $\alpha$ is related to the differentiation of nasopharyngeal carcinoma. In addition, the apoptosis inhibition pathway related to $\mathrm{PKC} \alpha$ has not been clarified and is worthy of further exploration. 
A

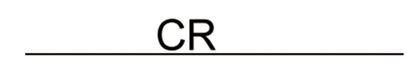

$\mathrm{PKC} \alpha$

Tubulin
B

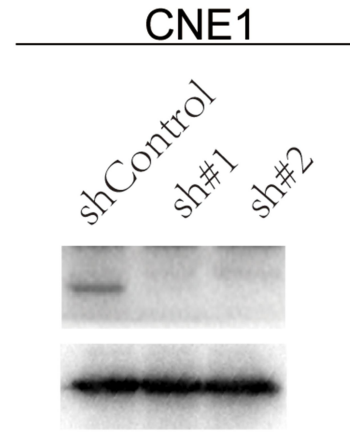

D
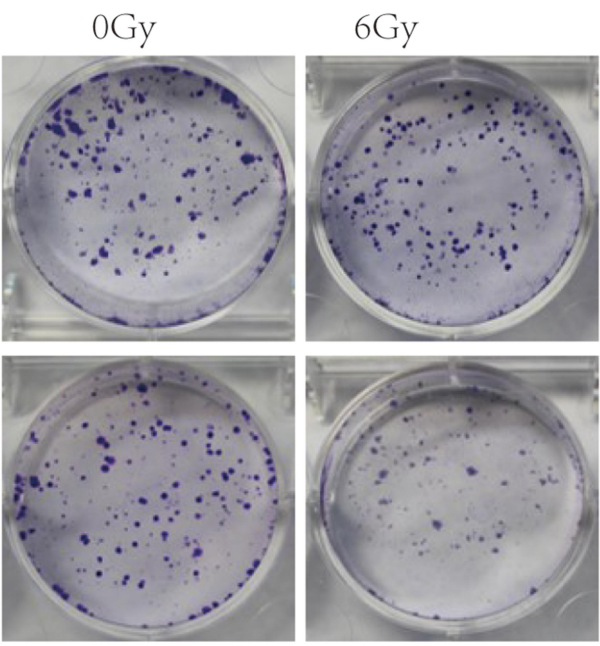

$\operatorname{shPKC} \alpha$

CNE1\#1
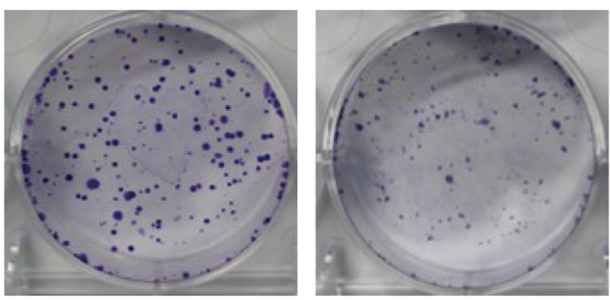

Figure 4 Inhibition of PKC $\alpha$ sensitizes NPC cells to radiation in vitro. (A) Western blotting of PKC $\alpha$ expression in CR cells after treatment with shControl, shPKC $\alpha$ \#I, or shPKC $\alpha$ \#2. (B) Western blotting of PKC $\alpha$ expression in CNEI cells after treatment with shControl, shPKC $\alpha \# I$, or shPKC $\alpha \# 2$. (C) Clonogenic assay for CR cells treated with shControl or shPKC $\alpha$ and later exposed to 0 or 6 Gy radiation $(n=3 ; P<0.05)$. (D) Clonogenic assay for $C N E I$ cells treated with shControl or shPKC $\alpha$ and later exposed to 0 or 6 Gy radiation $(n=3 ; P<0.05)$.

\section{Conclusions}

PKC $\alpha$ expression in increased in NPC tissues as well as in radio-resistant NPC cell lines. Moreover, a significant association was found between PKC $\alpha$ expression and the 5-year PFS and LRFS of NPC patients. Together, the findings of this study indicate that PKC $\alpha$ is a potential molecular marker of radio-resistance in
NPC, and thus, measurement of its expression level may aid in treatment planning for NPC patients. Moreover, our findings that loss of $\mathrm{PKC} \alpha$ expression rendered NPC cells more resistant to radiation therapy both in vitro and in vivo suggest that $\mathrm{PKC} \alpha$ is also a potential target for enhancing the radio-sensitivity of NPC. 
A

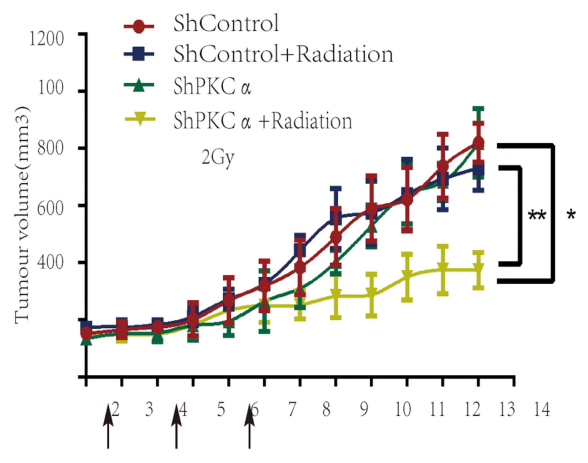

B

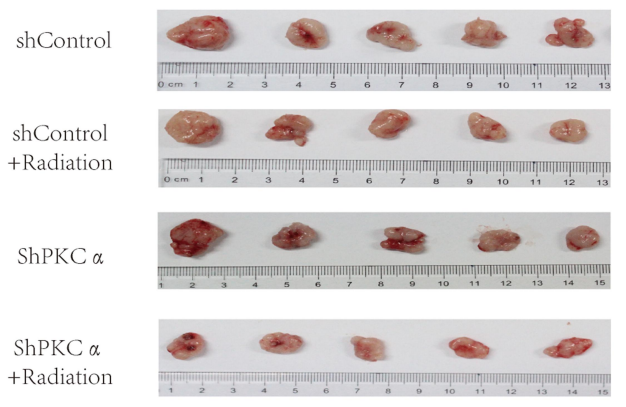

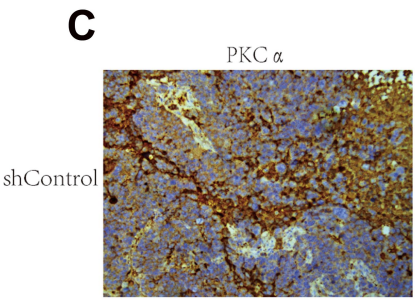

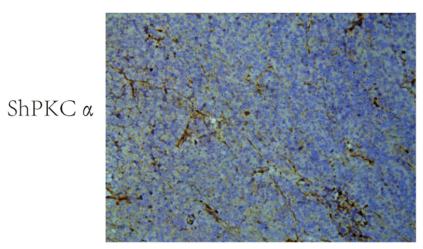

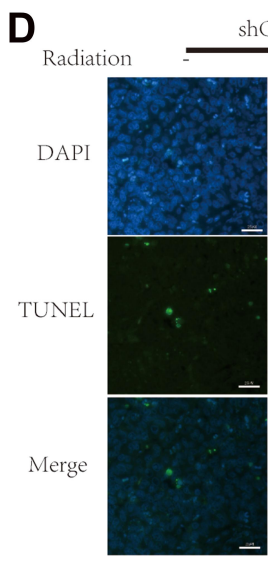
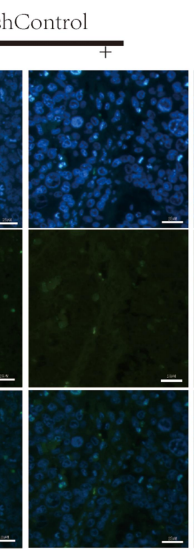

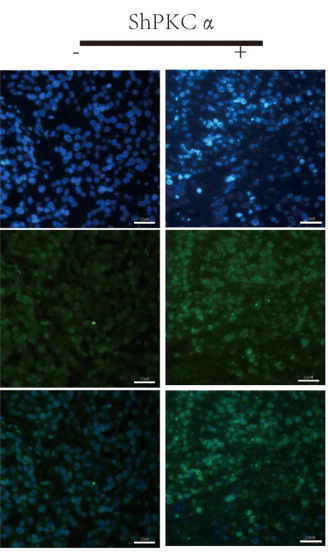

E

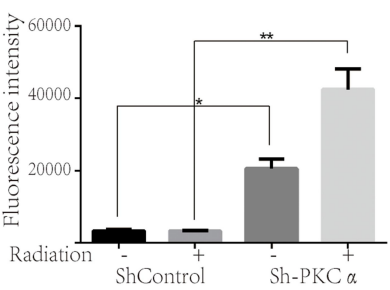

Figure 5 Inhibition of PKC $\alpha$ sensitized NPC xenografts to irradiation in vivo. (A) Growth curves of shControl-and shPKC $\alpha$-CR tumors with or without radiation treatment $(2 \mathrm{~Gy} \times 3$ fractions, $n=5)$. (B) Nude mouse tumor growth status $(n=5)$. (C) Immunohistochemical staining of PKC $\alpha$ in shControl- and shPKC $\alpha$-CR xenografts $($ scale bar $=25$ $\mu \mathrm{m})$. (D) Terminal deoxynucleotidyl transferase dUTP nick end labeling (TUNEL) staining of apoptotic cells in shControl- or shPKC $\alpha$-CR xenografts with or without radiation treatment (scale bar $=25 \mu \mathrm{m}$ ). (E) Quantitative analysis of TUNEL staining data in $(\mathbf{C})$. TUNEL-positive cells were counted in at least 8 fields on each slide. $* \mathrm{P}<0.05, * * \mathrm{P}<0.01$.

\section{Acknowledgment}

We thank Yang XH for his help with the experiments.

\section{Funding}

This work was supported by grants from the from the National Natural Science Foundation of China (81530084).

\section{Disclosure}

The authors declare that they have no competing interests. The authors alone are responsible for the content and writing of the paper.

\section{References}

1. Chua MLK, Wee JTS, Hui EP, Chan ATC. Nasopharyngeal carcinoma. Lancet. 2016;387:1012-1024. doi:10.1016/S0140-6736(15)00055-0
2. Wei WI, Sham JS. Nasopharyngeal carcinoma. Lancet. 2005;365:2041-2054. doi:10.1016/S0140-6736(05)66698-6

3. Tan LLY, Chua MLK. Discovering biomarkers of radioresistance in a radiosensitive cancer: a clinical paradox in nasopharyngeal carcinoma. Ann Transl Med. 2020;8:1284. doi:10.21037/atm-205405

4. Rosse C, Linch M, Kermorgant S, Cameron AJ, Boeckeler K, Parker PJ. PKC and the control of localized signal dynamics. Nat Rev Mol Cell Biol. 2010;11:103-112. doi:10.1038/nrm2847

5. Zhang J, Wang Y, Duan Y, et al. PKC $\alpha$ promotes local advancement via its dual roles in nasopharyngeal carcinoma. Acta Otolaryngol. 2017;137:662-667. doi:10.1080/00016489.2016.1269195

6. Sun Y, Li WF, Chen NY, et al. Induction chemotherapy plus concurrent chemoradiotherapy versus concurrent chemoradiotherapy alone in locoregionally advanced nasopharyngeal carcinoma: a Phase 3, multicentre, randomised controlled trial. Lancet Oncol. 2016;17:1509-1520. doi:10.1016/S1470-2045(16)30410-7

7. Lu J, Tang M, Li H, et al. EBV-LMP1 suppresses the DNA damage response through DNA-PK/AMPK signaling to promote radioresistance in nasopharyngeal carcinoma. Cancer Lett. 2016;380:191-200.

8. Hou Y, Zhu Q, Li Z, et al. The FOXM1-ABCC5 axis contributes to paclitaxel resistance in nasopharyngeal carcinoma cells. Cell Death Dis. 2017;8:e2659. doi:10.1038/cddis.2017.53 
9. He JJ, Li Z, Rong ZX, et al. m(6)A Reader YTHDC2 promotes radiotherapy resistance of nasopharyngeal carcinoma via activating IGF1R/ AKT/S6 signaling axis. Front Oncol. 2020;10:1166. doi:10.3389/ fonc. 2020.01166

10. Li G, Liu Y, Su Z, et al. MicroRNA-324-3p regulates nasopharyngeal carcinoma radioresistance by directly targeting WNT2B. Eur $J$ Cancer. 2013;49:2596-2607. doi:10.1016/j.ejca.2013.03.001

11. Fu S, Li Z, Xiao L, et al. Glutamine synthetase promotes radiation resistance via facilitating nucleotide metabolism and subsequent DNA damage repair. Cell Rep. 2019;28:1136-1143.e1134. doi:10.1016/j.celrep.2019.07.002

12. Tang J, Bouyer P, Mykoniatis A, Buschmann M, Matlin KS, Matthews JB. Activated PKC $\{$ delta $\}$ and PKC \{epsilon $\}$ inhibit epithelial chloride secretion response to cAMP via inducing internalization of the $\mathrm{Na}+-\mathrm{K}+-2 \mathrm{Cl}$ - cotransporter NKCC1. J Biol Chem. 2010;285:34072-34085. doi:10.1074/jbc.M110.137380

13. Zhang L, Xie B, Qiu Y, et al. Rab25-mediated EGFR recycling causes tumor acquired radioresistance. iScience. 2020;23:100997. doi:10.1016/j.isci.2020.100997

14. Rahimova N, Cooke M, Zhang S, Baker MJ, Kazanietz MG. The PKC universe keeps expanding: from cancer initiation to metastasis. Adv Biol Regul. 2020;78:100755. doi:10.1016/j.jbior.2020.100755

15. Antal CE, Hudson AM, Kang E, et al. Cancer-associated protein kinase $\mathrm{C}$ mutations reveal kinase's role as tumor suppressor. Cell. 2015;160:489-502. doi:10.1016/j.cell.2015.01.001

16. Parker PJ, Brown SJ, Calleja V, Chakravarty P, Cobbaut M. Equivocal, explicit and emergent actions of PKC isoforms in cancer. Nat Rev Cancer. 2021;21:51-63. doi:10.1038/s41568-020-00310-4
17. Wang Q, Zhang T, Chang X, et al. Targeting Opsin4/Melanopsin with a novel small molecule suppresses PKC/RAF/MEK/ERK signaling and inhibits lung adenocarcinoma progression. Mol Cancer Res. 2020;18:1028-1038. doi:10.1158/1541-7786.MCR-19-1120

18. Deka SJ, Trivedi V. Potentials of PKC in cancer progression and anticancer drug development. Curr Drug Discov Technol. 2019;16:135-147. doi:10.2174/1570163815666180219113614

19. Lau WM, Teng E, Huang KK, et al. Acquired resistance to FGFR inhibitor in diffuse-type gastric cancer through an AKT-independent PKC-mediated phosphorylation of GSK3 $\beta$. Mol Cancer Ther. 2018;17:232-242. doi:10.1158/1535-7163.MCT-17-0367

20. Bluwstein A, Kumar N, Léger K, et al. PKC signaling prevents irradiation-induced apoptosis of primary human fibroblasts. Cell Death Dis. 2013;4:e498. doi:10.1038/cddis.2013.15

21. Scott JG, Berglund A, Schell MJ, et al. A genome-based model for adjusting radiotherapy dose (GARD): a retrospective, cohort-based study. Lancet Oncol. 2017;18:202-211. doi:10.1016/S1470-2045(16)30648-9

22. Domínguez-García S, Geribaldi-Doldán N, Gómez-Oliva R. A novel PKC activating molecule promotes neuroblast differentiation and delivery of newborn neurons in brain injuries. Cell Death Dis. 2020;11:262. doi:10.1038/s41419-020-2453-9

23. Zhao H, Li M, Ouyang Q, Lin G, Hu L. VEGF promotes endothelial cell differentiation from human embryonic stem cells mainly through PKC- $\varepsilon / \eta$ pathway. Stem Cells Dev. 2020;29:90-99. doi:10.1089/ scd.2019.0172

24. Nakura A, Higuchi C, Yoshida K, Yoshikawa H. PKC $\alpha$ suppresses osteoblastic differentiation. Bone. 2011;48:476-484. doi:10.1016/j. bone.2010.09.238

\section{Publish your work in this journal}

Cancer Management and Research is an international, peer-reviewed open access journal focusing on cancer research and the optimal use of preventative and integrated treatment interventions to achieve improved outcomes, enhanced survival and quality of life for the cancer patient.
The manuscript management system is completely online and includes a very quick and fair peer-review system, which is all easy to use. Visit http://www.dovepress.com/testimonials.php to read real quotes from published authors. 